\title{
Effets anti-prolifératif et anti-inflammatoire des gluco-corticoïdes par inhibition des complexes Jun-Fos
}

Les glucocorticoïdes se comportent comme des inhibiteurs de la prolifération de nombreuses cellules et comme de puissants antiinflammatoires. Les mécanismes de ces actions sont très imparfaitement connus. Il semble probable, quoique non définitivement prouvé, qu'une des voies en cause est celle des lipocortines, inhibiteurs de la phospholipase A2 et donc de la synthèse des intermédiaires de l'inflammation que sont les icosanoïdes (dérivés de l'acide arachidonique parmi lesquels on trouve les prostaglandines, les leucotriènes, le PAF-aceter et les thromboxanes) [1, 2]. Il existe aussi toute une série de gènes codant pour des métalloprotéases (collagénase, stromélysine) impliquées dans la dégradation des protéines du collagène et des membranes basales, gènes dont la transcription est précocement induite sous l'effet de cytokines médiatrices de l'inflammation telles que l'interleukine 1 et le tumor necrosis factor (TNF). Les enzymes jouent probablement un rôle très important dans certains processus inflammatoires tels que la destruction cartilagineuse, osseuse et tendineuse observée dans l'arthrite rhumatoïde. Les régions de régulation des gènes de la collagénase et de la stromélysine contiennent des sites de fixation du complexe protéique AP1 qui est principalement composé d'homo-dimères constitués de deux protéines Jun, de faible affinité, ou d'hétéro-dimères comprenant une sous-unité Jun et une sous-unité Fos, de haute affinité. La zone de dimérisation est maintenant bien connue : elle correspond à une structure particulière comportant des leucines tous les 7 acides aminés, ce qui les amène à se trouver sur un même côté d'une hélice $\alpha$, formant une sorte de "fermeture à glissière de leucines" ou leucine zipper. Les sous-unités interagissent par l'intermédiaire de liaisons hydrophobes entre leurs régions à leucine zipper.

Des résultats récents avaient montré qu'au niveau du gène de la proliférine, activé par les facteurs de croissance et les esters de phorbol, le site AP1 était tout à la fois indispensable à l'activation transcriptionnelle et à la répression par les glucocorticoïdes [3]. Ces données ont conduit deux équipes nord-américaines associées, l'une de La Jolla (CA, USA), dirigée par Michael Karin, et l'autre de Montréal, dirigée par Jacques Drouin, à tester l'hypothèse selon laquelle une interférence entre le récepteur des glucocorticoïdes et les protéines Jun et Fos était responsable des effets de ces hormones sur la transcription des gènes de métalloprotéases [4]. Leurs expériences ont comporté la transfection de cellules avec tout à la fois un vecteur d'expression commandant la synthèse du récepteur et une construction contenant un gène test sous le contrôle du promoteur du gène de la collagénase. L'expression du gène test était d'autant plus inhibée par les glucocorticoïdes que la quantité de récepteur synthétisée était plus grande. Dans d'autres expériences, les auteurs devaient démontrer que le récepteur contractait des interactions spécifiques avec les protéines Jun et Fos, des complexes récepteur/Jun et récepteur/Fos pouvant être isolés après établissement in vitro de liaisons covalentes entre ces molécules à l'aide de réactifs spécifiques de groupes sulfhydriles. Ces complexes semblent incapables de se fixer à la fois sur les motifs spécifiques des récepteurs (c'est-à-dire les éléments GRE, glucocorticoid response elements) et sur les sites AP1. Seule la région du récepteur impliquée dans sa liaison à l'ADN est indispensable à la formation des complexes, et des arguments indirects semblent indiquer que c'est la région des leucine zippers, à l'extrémité carboxyterminale de Jun et Fos, qui intervient dans la fixation au récepteur. Le modèle qui émerge de ces expériences est donc celui d'une interaction entre les motifs de liaison à l'ADN de deux facteurs de transcription agissant en sens inverse, le récepteur des glucocorticoïdes et les composants des complexes AP1. Par ce mécanisme, ces protéines de régulation se contrôlent l'une l'autre ; le récepteur des glucocorticoïdes, activé par la fixation de l'hormone, inhibe 


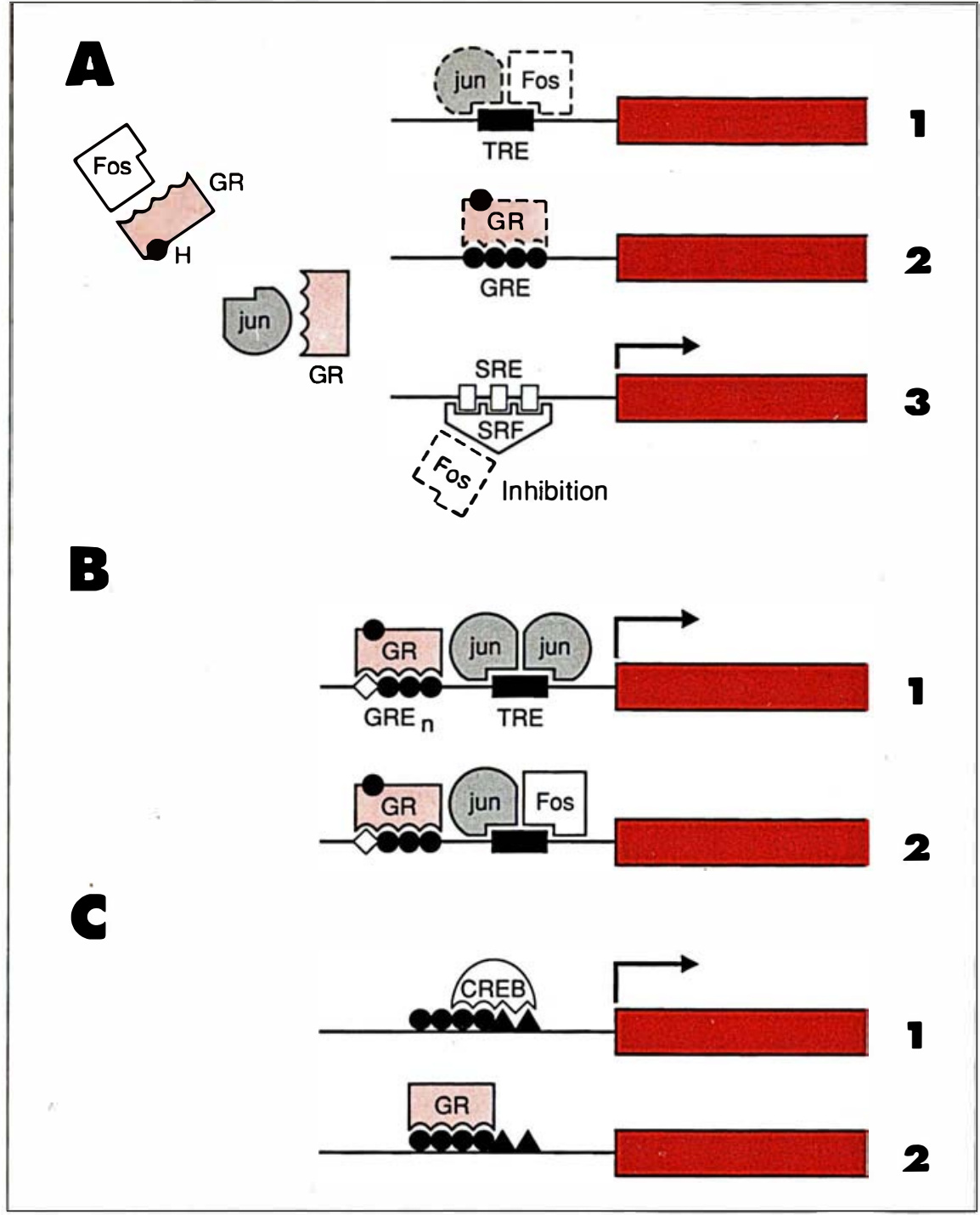

Figure 1. Différents modèles de régulation négative de la transcription des gènes par les glucocorticoïdes et leur récepteur. TRE : TPA response element, site de fixation de complexes AP1; GRE : glucose response element, site de fixation du récepteur des glucocorticoïdes (GR) ; GRE ${ }_{n}$ : GRE négatif; SRE : serum response element, site de fixation du facteur protéique SRF ; CRE : cyclic AMP response element, site de fixation du facteur protéique CREB; $H$ : hormone (glucocorticoïdes). Les flèches partant du début du gène (rectangle rouge) indiquent l'activité transcriptionnelle. A) Modèles présentés dans les références 4, 5 et 7. La formation des complexes entre GR et Fos ou Jun rend ces trois protéines indisponibles pour exercer leurs effets biologiques: stimulation transcriptionnelle des gènes à TRE par le complexe Jun-Fos (1), des gènes à GRE par le GR (2), inhibition des gènes à SRE par Fos (3). En pointillé est représenté l'agencement des facteurs lorsqu'ils ne sont pas complexés. B) Modèle présenté dans la référence 8. L'interaction au niveau d'un GRE $E_{n}$ dégénéré et d'un TRE proche de GR et des complexes AP1 dépend de la nature de ces derniers : activation s'il s'agit d'un homo-dimère JunJun (1), inhibition dans le cas d'un hétéro-dimère Fos-Jun (2). C) Modèle présenté dans la référence 11. La fixation de l'activateur CREB et de GR étant mutuellement exclusive, les hormones glucocorticoïdes inhibent la transcrip- l'action des produits d'oncogènes Fos et Jun et ceux-ci, lorsqu'ils sont fortement exprimés, peuvent inhiber l'action des glucocorticoïdes (figure 1). Un modèle assez voisin a été proposé par d'autres équipes. Celle de Ronald Evans, de La Jolla (CA, USA), utilisant elle aussi le promoteur du gène de la collagénase et son site AP1, aboutit pratiquement aux mêmes conclusions que Karin et Drouin [5]. Étudiant toujours l'inhibition du gène de la collagénase par les glucocorticoïdes, les chercheurs allemands du laboratoire de Peter Herrlich (Karlsruhe, RFA) apportent quelques résultats complémentaires intéressants et différent légèrement des équipes nordaméricaines quant au modèle précis de l'interaction entre le récepteur des glucocorticoïdes et le complexe AP1 qu'il suggèrent. Ces chercheurs montrent en effet que la concentration de glucocorticoïdes susceptible d'inhiber la transcription d'un gène test contrôlé par des sites AP1 est nettement inférieure aux concentrations nécessaires pour stimuler la transcription des gènes répondant à ces hormones [6]. le RU 486 est un puissant inhibiteur de l'action des glucocorticoïdes qui se lie au récepteur et n'empêche pas la fixation de ce dernier au GRE, mais bloque son potentiel d'activation transcriptionnelle. Cette anti-hormone s'oppose à l'inhibition, par le récepteur, du gène test contrôlé par les sites $\mathrm{AP} 1$, à des doses là encore inférieures à celles capables d'inhiber des gènes stimulés par les cocorticoïdes. La divergence entre les résultats de Jonat $e t$ al. [6] et ceux des autres équipes réside dans l'aptitude, supposée par l'équipe allemande et déniée par les autres, qu'aurait le récepteur lié au GRE de fixer le complexe Jun-Fos.

Le groupe de Rolf Müller, à Marburg (RFA), a étudié particulièrement les interactions entre Fos et le récepteur et conclu que ces deux protéines s'inhibaient mutuellement, quelle que soit leur action sur la transcription des gènes [7]. En d'autres termes, lorsque Fos est inhibiteur (par exemple de la transcription de son propre gène), cette inhibition est levée par les glucocorticoïdes (figure 1). Contrairement, cependant, aux résultats de l'équipe de Karin et de Drouin [4], Lucibello et 
al. montrent que c'est la région aminoterminale de Fos qui semble interagir avec la région de liaison à l'ADN du récepteur des glucocorticoïdes, assignant donc une fonction à cette région dont on ignorait jusqu'alors la signification [7]. Il existe, à côté de la protéine c-Fos étudiée jusqu'ici, d'autres protéines assez similaires, dont Fos B qui diffère de c-Fos par son extrémité aminoterminale: le récepteur des glucocorticoïdes est, probablement de ce fait, incapable de former un complexe avec Fos B [7].

Contrairement aux quatre articles commentés ci-dessus, dans lesquels l'inhibition par le récepteur des glucocorticoïdes de l'action biologique de Fos et de Jun ne nécessite aucun site de type GRE (glucocorticoid response element), les résultats présentés par l'équipe de Keith R. Yamamoto (San Francisco, CA, USA) supposent l'intervention d'un GRE négatif à faible affinité adjacent à un site de fixation du complexe AP1 [8]. Ces auteurs montrent que la séquence régulatrice du gène de proliférine contient, à côté du site $\mathrm{AP} 1$, un site GRE à faible affinité pour le récepteur. Les conclusions des expériences menées dans ce travail sont que l'interaction sur la séquence d'ADN d'un récepteur lié à l'hormone et d'un dimère Jun-Fos aboutit à l'inhibition de la stimulation provoquée par l'hétéro-dimère seul alors que l'interaction récepteur/dimère JunJun renforce la stimulation due à l'homo-dimère seul (figure 1). Ces conclusions portant sur un autre modèle que ceux utilisés par les autres équipes ne sont pas forcément contradictoires avec les données résumées précédemment. Elles peuvent fournir un schéma de portée générale pour expliquer le mode d'action de ces "GRE négatifs" décrits pour plusieurs gènes $[9,10]$ et ayant en commun d'être des éléments de séquence dégénérés par comparaison aux GRE activateurs fixant le récepteur avec une grande affinité.

Un troisième mécanisme d'inhibition transcriptionnelle par les glucocorticoïdes a également été rapporté il y a quelques années ; il met en jeu le chevauchement entre un GRE à haute affinité et un site de fixation d'un activateur transcriptionnel, par exemple la protéine CREB. (cyclic AMP response element binding protein), responsable de l'activation transcriptionnelle de certains gènes par l'AMP cyclique. $\mathrm{La}$ fixation du récepteur et de CREB étant mutuellement exclusive, les glucocorticoïdes inhibent la transcription du gène en provoquant le déplacement de l'activateur (figure 1) [11]. Peut-être est-ce un mécanisme de ce type qui explique l'antagonisme entre la vitamine $\mathrm{D}$, se fixant à un récepteur de la même famille que celui des glucocorticoïdes, et le complexe Jun-Fos pour l'activation du gène de l'ostéocalcine. Ce gène contient dans son promoteur un VDRE (vitamin $D$ response element) $(\mathrm{m} / \mathrm{s}$ $n^{\circ} 3$, vol. $6, p$. 307) qui fixe non seulement le récepteur de la vitamine $\mathrm{D}$, mais aussi celui de l'acide rétinoïque, ces deux protéines liées à leur ligand se comportant comme des activateurs transcriptionnels. Il semble qu'il existe, au sein du VDRE, une séquence de type AP1 liant les dimères Jun-Fos (et peut-être Jun-Jun), la fixation du complexe AP1 et des récepteurs hormonaux étant, là encore, mutuellement exclusive [12]. Ce résumé de quelques mécanismes de l'action inhibitrice des glucocorticoïdes sur d'autres facteurs transcriptionnels nous permet de souligner une caractéristique de l'évolution actuelle des conceptions concernant la régulation de l'expression des gènes. L'interaction élémentaire entre des protéines et des séquences particulières d'ADN n'est qu'un des mécanismes - incontournable - en cause, la diversité et la souplesse d'adaptation des processus régulateurs mettant en jeu de nombreux phénomènes de coopération, d'oligomérisation et de modifications mutuelles des facteurs de transcription. L'inactivation biologique par le récepteur des glucocorticoïdes lié à ses ligands de ces médiateurs transcriptionnels de l'inflammation et de la prolifération que sont Jun et Fos pourrait constituer l'un des modes d'action principaux des glucocorticoïdes, d'autant plus qu'elle survient à faible concentration de l'hormone

\section{Note ajoutée aux épreuves}

L'inhibition transcriptionnelle par les glucocorticoïdes semble pouvoir utiliser encore d'autres mécanismes que ceux résumés ci-dessus. Une équipe new-yorkaise vient en effet de démontrer que le récepteur des glucocorticoïdes lié à l'hormone pouvait interférer avec les régions les plus proximales du promoteur du gène de l'interleukine 6 (IL-6) [13]. Cette cytokine et les glucocorticoïdes ont des relations complexes : dans l'hypothalamus, il semble que IL-6 provoque une libération du CRF (corticotropin releasing factor) et donc indirectement d'ACTH par l'hypophyse et de glucocorticoïdes par la surrénale. Au niveau du foie, IL-6 et glucocorticoïdes vont coopérer pour induire la transcription des gènes de la phase aiguë de la réaction inflammatoire (codant, par exemple, pour le fibrinogène, l'haptoglobine et bien d'autres protéines sériques dont la concentration est élevée dans les réactions inflammatoires). Cette réaction va néanmoins être autolimitée, car les glucocorticoïdes vont rapidement bloquer la transcription du gène $I L-6$. Cette inhibition semble liée à la fixation du complexe hormone/récepteur au niveau du promoteur et du enhancer du gène $I L-6$, de manière mutuellement exclusive avec des activateurs transcriptionnels. Notamment, le complexe se fixe au niveau de la TATA box, site de fixation du facteur TFIID (transcriptional factor $D$ des promoteurs dépendant de l'ARN polymérase II) et du site d'initiation de la transcription. Ces sites d'initiation se ressemblent dans de nombreux gènes, reproduisant une séquence In $r$ (Initiator) de type CTCATTCT. Ils fixent probablement un facteur transcriptionnel dont les liens avec TFIID restent inconnus. 


\section{FLASH}

\section{LE GĖNE p53, GĖNE DE PRÉDISPOSITION AU CANCER}

\section{RÉFÉRENCES}

1. Rothhut B, Russo-Marie F. Les lipocortines. médecine/sciences $1987 ; 3$ : 282-7.

2. Chap H. Isocanoïdes et phospholipases. médecine/sciences $1988 ; 4: 6-7$.

3. Mordacq JC, Linzer DIH. Colocalization of elements required for phorbol ester stimulation and glucocorticoid repression of proliferin gene expression. Gene Dev 1989 ; 3 : 760-9.

4. Yang-Yen HF, Chambard JC, Sun YL, et al. Transcriptional interference between c-Jun and glucocorticoid receptor due to the mutual inhibition of DNA binding activity mediated by direct protein-protein interaction. Cell 1990 ; 62 : 1205-15.

5. Schüle R, Rangarajan P, Kliewer S, et al. Functional antagonism between oncoprotein c-Jun and the glucocorticoid receptor. Cell $1990 ; 62$ : 1217-26.

6. Jonat C, Rahmsdorf HJ, Park KK, et al. Antitumor promotion and antiinflammation : down-modulation of AP1 (Fos-Jun) activity by glucocorticoid hormone. Cell 1990; 62 : 1189-204.

7. Lucibello FC, Slater EP, Jooss KU, Beato M, Muller R. Mutual transrepression of Fos and the glucocorticoid receptor: involvement of a functional domain in Fos which is absent in Fos B. Embo J $1990 ; 9$ : 2827-34.

8. Diamond MI, Miner JN, Yoshinaga SK, Yamamoto KR. Transcription factor interactions : selectors of positive or negative regulation from a single DNA element. Science $1990 ; 249$ : 1266-71.

9. Drouin J, Trifiro MA, Plante RK, Nemer M, Ericksson P, Wrange O. Glucocorticoid receptor binding to a specific DNA sequence is required for hormone dependent inhibition of proopiomelanocortin gene transcription. Mol Cell Biol 1989 ; 9 : 5305-14.

10. Sakai DD, Helms S, Carlstedt-Duke J, Gustaf sson JA, Rottman FM, Yamamoto KR. Hormone-mediated repression : a negative glucocorticoid response element from the bovine prolactin gene. Gene Dev 1988 ; 2 : 1144-54.

11. Akerblom IE, Slater EP, Beato $M$, Baxter JD, Mellon PL. Negative regulation by glucocorticoids through interference with cAMP responsive enhancer. Science 1988 ; $241: 350-3$.

12. Schüle $R$, Umesono $K$, Mangelsdorf $D$, Bolado J, Pike JW, Evans RM. Jun-Fos and receptors for vitamins $A$ and $D$ recognize a common response element in the human osteocalcin gene. Cell 1990 ; 61 . 497-504.

13. Ray A, LaForge KS, Seghal PB. On the mechanism for efficient repression of the interleukine 6 promoter by glucocorticoids : enhancer, TATA box and RNA start site (Inr motif) occlusion. Mol Cell Biol 1990 ;
Le syndrome de Li Fraumeni (LFS) représente l'agrégation familiale d'un large spectre de cancers incluant des carcinomes mammaires, des sarcomes des tissus mous, des ostéosarcomes, des leucémies et des corticosurrénalomes. Ces diverses tumeurs apparaissent à un âge inhabituellement précoce et il n'est pas rare qu'un même individu soit atteint de multiples tumeurs primaires. L'analyse de ségrégation démontre que l'existence d'un gène autosomal dominant peut rendre compte de la distribution familiale des cancers et ce modèle prédit que $90 \%$ des porteurs de ce gène seront atteints d'un cancer avant 70 ans.

L'inactivation du gène suppresseur de tumeur p53 par déletion ou mutation été observée dans un grand nombre de tumeurs sporadiques du sein, des os ou des tissus mous, qui sont les tissus affectés par les tumeurs dans les familles LFS. S. Friend, et F. Li leurs collaborateurs (Boston, MA, USA) [1] faisant I'hypothèse que le gène p53 pouvait représenter un gène de susceptibilité ont donc recherché des mutations constitutionnelles de ce gène chez des individus sains ou atteints de cancers appartenant à cinq familles LFS indépendantes. Ces mutations ont été recherchées par séquençage d'un fragment du gène (amplifié par PCR) où sont localisées la plupart des mutations observées dans les tumeurs sporadiques (exons 5 à 8 inclus). Les individus affectés de tumeurs sont tous hétérozygotes pour p53, ils portent un allèle sauvage et un allèle muté sur l'un des quatre codons 245, 248, 252 ou 258, mutations inactivantes déjà reconnues dans les tumeurs sporadiques. Chez l'un de ces individus, I'analyse de la tumeur révèle l'allèle muté et la perte de l'allèle sauvage conduisant à une perte complète de l'activité suppresseur. Parmi les individus sains certains sont hétérozygotes et présentent vraisemblablement un risque plus élevé de cancers. Les individus homozygotes pour deux allèles normaux de p53 sont tous sains.

Ces résultats démontrent que les mutations du gène p53 interviennent non seulement au niveau somatique dans certains cancers mais également au niveau des cellules germinales, supports de leur transmission héréditaire. La présence constitutive d'un allèle muté représente pour un large spectre de tissus un facteur de risque de cancer. Le développement d'un diagnostic moléculaire permettant de détecter ces mutations germinales de p53 permet d'envisager l'exploration systématique des individus sains de familles LFS. L'identification des porteurs d'allèles mutés affectés d'un haut risque de cancer facilitera les stratégies de prévention ; alors que celle des non porteurs représentera pour ceuxci un réconfort certain.

Jean Feunteun Institut Gustave Roussy Villejuif

1. Malkin D. Li FP. Strong LC, et al. Germ line p53 mutations in a familial syndrome of breast cancer, sarcomas, and other neoplasms. Science 1990, 250: 1233-8. 